\title{
Low-Dose Ethanol Has Impacts on Plasma Levels of Metabolites Relating to Chronic Disease Risk in SAMP8 mice
}

\author{
Churan Fu ${ }^{1}$, Yongshou YANG ${ }^{1}$, Thanutchaporn KumRungSEe ${ }^{1}$, Akiko Kimoto ${ }^{2}$, \\ Hanae IZU $^{3}$ and Norihisa KATO ${ }^{1, *}$ \\ ${ }^{1}$ Graduate School of Integrated Sciences for Life, Hiroshima University, \\ Higashi-Hiroshima 739-8528, Japan \\ ${ }^{2}$ Faculty of Human Ecology, Yasuda Women's University, Asaminami-ku, Hiroshima 731-0153, Japan \\ ${ }^{3}$ Quality and Evaluation Research Division, National Research Institute of Brewing, \\ Higashi-Hiroshima 739-0046, Japan
}

(Received April 8, 2020)

\begin{abstract}
Summary The effects of low-dose alcohol on experimental animals are unclear. This study examined plasma metabolites in senescence-accelerated mice 8 (SAMP8) given lowdose ethanol, and compared them with aging progress and skeletal muscle strength. Male SAMP8 mice (10-wk-old) were given drinking water containing $0 \%$ (control), $1 \%, 2 \%$, or $5 \%(\mathrm{v} / \mathrm{v})$ ethanol for $14 \mathrm{wk}$. Compared with the control group, only mice who consumed $1 \%$ ethanol experienced a lower senescence score at 18 and $23 \mathrm{wk}$, as well as an increased limb grip strength at $21 \mathrm{wk}$. Plasma metabolites of control, $1 \%$ and $2 \%$ ethanol groups were analyzed by capillary electrophoresis-time-of-flight mass spectrometry (CE-TOF/MS). Among the 7 metabolites affected by ethanol, notewhorthy is the positive association of the ethanol levels in drinking water with the levels of $\alpha$-ketoglutarate (antioxidant and anti-inflammatory metabolite) and hippurate (antioxidant and microbial co-metabolite) $(p<0.05)$. Intriguingly, the levels of 2-hydroxyisobutyrate (the biomarker of energy metabolism and microbial co-metabolite) were higher in the $1 \%$ ethanol group $(p<0.05)$, but not in the $2 \%$ ethanol group as compared to the control. Furthermore, the levels of some of the metabolites affected were correlated with some variables in the grading score of senescence and muscle strength. This study provides a novel insight into how low-dose ethanol in SAMP8 mice modulates the levels of circulating metabolites relating to chronic disease risk.
\end{abstract}

Key Words low-dose ethanol, senescence-accelerated mice, antioxidant, anti-inflammatory metabolite, microbial co-metabolite

In 1991, Marmot and Brunner (1) reported a U- or J-shaped relationship between alcohol (i.e., ethanol) consumption and disease risk: moderate consumption was associated with lower risks of chronic diseases such as cardiovascular and cancer, whereas high consumption was associated with a higher risk. Interestingly, minimal (or low dose) consumption has been reported by several epidemiological studies to have a beneficial effect on overall health $(2,3)$. However, there are epidemiological studies to report no beneficial effects of lowdose alcohol $(4,5)$. Thus, the effects of low-dose alcohol remain controversial.

Given the limitations of epidemiological studies, the direct effects of low-dose ethanol on health cannot be confirmed and, thus, remain controversial. In humans, laboratory investigations evaluating the impact of alcohol on an individual's overall health is challenging be-

\footnotetext{
* To whom correspondence should be addressed.

E-mail: nkato@hiroshima-u.ac.jp

Abbreviations: CE, capillary electrophoresis; CE-TOF/MS, capillary electrophoresis-time-of-flight mass spectrometry; DMG, N,N-dimethylglycine; ESI, electrospray ionization; 2HIB, 2-hydroxyisobutyrate; IBD, inflammatory bowel disease; SAMP8, senescence-accelerated mice 8 .
}

cause of confounding factors such as socioeconomic status, ethnicity, medical history, and frequency and type of alcoholic beverages consumed. Therefore, it is necessary to conduct animal experiments to clarify the effects of low-dose ethanol. Martin et al. (6) reported that rats consuming water with $1 \%$ ethanol experienced a reduction in total cholesterol and triglyceride levels, thereby decreasing the risk of cardiovascular disease. Osaki et al. (7) found that consumption of $1 \%$ ethanol in drinking water $\left(\sim 0.3 \mathrm{~g} \cdot \mathrm{kg}^{-1} \cdot \mathrm{d}^{-1}\right)$ improved liver function in rats fed a high-fat diet, whereas intake of $2 \%$ ethanol had only a minor effect. Kimoto et al. (8) subsequently reported that in senescence-accelerated mice 8 (SAMP8), being widely used in brain aging study, consumption of $1 \%$ ethanol suppressed senescence grading scores and markedly increased brain mRNA levels of alcohol dehydrogenase 1 (an enzyme responsible for the degradation of ethanol and lipid peroxidation products), whereas $2 \%$ ethanol does not. Moreover, in our recent study, we found that consumption of $1 \%$ ethanol in drinking water $(0.4-0.5$ $\mathrm{g} \cdot \mathrm{kg}^{-1} \cdot \mathrm{d}^{-1}$ ) suppressed colon tumorigenesis and lowered the intestinal level of Clostridium leptum (immunosuppressor) in rats administered 1,2-dimethylhydrazine, 
whereas $2 \%$ ethanol did not (9). The results of another previous study suggest that low ethanol consumption $\left(0.7 \mathrm{~g} \cdot \mathrm{kg}^{-1} \cdot \mathrm{d}^{-1}\right)$ protects against cerebral ischemia/ reperfusion injury by suppressing postischemic inflammation via upregulation of cystathionine $\gamma$-lyase that, in turn, produces hydrogen sulfide (an anti-inflammatory and antioxidant metabolite at low concentrations) (10). Thus, these experimental studies suggest that intake of low-dose alcohol has beneficial effects on health. In contrast, there is some evidence that lowdose ethanol consumption leads to adverse effects in experimental animals. Melchior et al. have reported that low doses of ethanol $\left(0.5\right.$ or $\left.1.5 \mathrm{~g} \cdot \mathrm{kg}^{-1} \cdot \mathrm{d}^{-1}\right)$ impair working memory in mice (11). Moreover, Sakazaki et al. have reported that low-dose ethanol $\left(\sim 1 \mathrm{~g} \cdot \mathrm{kg}^{-1} \cdot \mathrm{d}^{-1}\right)$ aggravates allergic dermatitis in mice (12).

Given that the effects of low-dose ethanol are controversial and incompletely understood, our primary aim was to analyze the levels of plasma metabolites in SAMP8 mice administered low-dose ethanol by capillary electrophoresis-time-of-flight mass spectrometry (CE-TOF/MS) in the present study. As a secondary aim, we examined the correlations between the levels of metabolites and senescence and muscle strength in SAMP8 mice, based on preliminary discovery showing low-dose ethanol ( $1 \%$ ethanol in drinking water) increases limb grip strength (Izu et al., unpublished results), although high-dose ethanol is reported to cause skeletal muscle damage or alcoholic myopathy through mechanisms involving oxidative stress (13).

\section{MATERIALS AND METHODS}

Animal experiments. Five-week-old male SAMP8 mice (Japan SLC, Shizuoka, Japan) were kept in plastic cages ( 5 mice per cage) in a controlled environment (temperature $22 \pm 2{ }^{\circ} \mathrm{C}$, lights on from 00:00 to 12:00). Mice were given commercial food pellets (MF, Oriental Yeast, Tokyo, Japan) and water ad libitum. After $6 \mathrm{wk}$ of acclimation, the mice were divided into 4 groups $(n=10$ per group) given deionized water containing $0 \%$ (control), $1 \%, 2 \%$, or $5 \%(\mathrm{v} / \mathrm{v})$ ethanol ad libitum for $14 \mathrm{wk}$. Body weight was measured once every $2 \mathrm{wk}$, and food and drinking water intake were measured twice per week. On the day of dissection, food and drinking water were removed from 8:00 h. The mice were sacrificed (between 13:00 and 15:00) by decapitation after anesthesia with $3-4 \%$ isoflurane gas. All procedures followed the Guide for the Care and Use of Laboratory Animals (National Research Institute of Brewing, Animal Care Committee) and were approved by the Animal Care Committee of the National Research Institute of Brewing, Japan (Ethical approval No. 28-1).

Senescence grading. A grading system (8) was used to quantify and evaluate the degree of senescence. This system comprises 11 items that can be divided into 4 categories: behaviors (reactivity and passivity), skin and hair (glossiness, coarseness, hair loss, and skin ulcers), eyes (periophthalmic lesions, cataract, corneal ulcer, and corneal opacity), and spine (lordokyphosis). Each item was scored weekly by careful inspection. The total grading score was the sum of all 11 items scores.

Grip strength test. A grip strength test was performed from 10:00-12:00 h by using a grip strength meter tester (GPM-100B, Melquest, Toyama, Japan) to quantify the muscular strength of the limbs and forearms of mice (14). Briefly, each mouse was lifted by its tail and moved horizontally toward the grip bar (used to test the forearms) or grid (used to test all 4 limbs) of the meter until the animals' front legs or all 4 limbs reached and firmly grabbed the testing grip or grid, respectively. The peak force strength of the forearms or all limbs was measured and recorded while the mouse was slowly pulled from the meter at a constant speed.

Plasma CE-TOF/MS analysis. During dissection, blood was collected from the control group ( $0 \%$ ethanol), $1 \%$ ethanol, and $2 \%$ ethanol groups ( $n=8$ per group) into EDTA-containing tubes (JIS T3233, Becton, Dickinson and Company, Fukushima, Japan). Plasma was obtained by centrifugation at $1,300 \times g$ for $15 \mathrm{~min}$. Owing to financial limitations, 2 plasma samples of equal volume within the same group were randomly pooled into a single sample for metabolomics analysis. Thus, a total of 12 plasma samples (4 pooled plasma samples each from the control, $1 \%$ ethanol, and $2 \%$ ethanol groups) were subjected to metabolomics analysis (Human Metabolome Technologies Inc., Tsuruoka, Japan) according to the manufacturer's instructions (15). Plasma samples in the $5 \%$ ethanol group were not analyzed because there appeared to be no notable difference in the data of total senescence score and grip strength between the $2 \%$ and $5 \%$ ethanol groups. Briefly, $50 \mu \mathrm{L}$ serum sample was mixed into $450 \mu \mathrm{L}$ adjusted methanol in order to obtain $10 \mu \mathrm{M}$ internal standard. Then, $500 \mu \mathrm{L}$ chloroform and $200 \mu \mathrm{L}$ Milli-Q water were added and mixed, followed by centrifugation at $2,300 \times g$ at $4^{\circ} \mathrm{C}$ for $5 \mathrm{~min}$. The supernatant was ultrafiltered using a 5 kDa centrifugal filter unit (Millipore, Billerica, MA, USA) at $9,100 \times g$ at $4^{\circ} \mathrm{C}$ for $120 \mathrm{~min}$, followed by evaporation to dryness. Metabolomics analysis was performed using a capillary electrophoresis-time-of-flight mass spectrometry (CE-TOF/MS) system (Agilent Technologies, Waldbronn, Germany), and separation was performed in fused-silica capillaries $(50 \mu \mathrm{m}$ i.d. $\times 80 \mathrm{~cm}$ total length). For cationic metabolites, the MS conditions were as follows: sample injection, pressure injection, 50 mbar for $10 \mathrm{~s}$; CE voltage: positive, $27 \mathrm{kV}$; ionization: electrospray ionization (ESI) in positive mode; capillary voltage: 4,000 V; scan range: $\mathrm{m} / \mathrm{z}$ 50-1,000. For anionic metabolites, the MS conditions were as follows: sample injection, pressure injection, 50 mbar for $10 \mathrm{~s}$; CE voltage: positive, $30 \mathrm{kV}$; ionization: ESI in negative mode; capillary voltage: $3,500 \mathrm{~V}$; scan range: $\mathrm{m} / \mathrm{z}$ 50-1,000.

Statistical analysis. Data were analyzed by Dunnett's test. The significant level was set at $p<0.05$. Correlation was analyzed by measure of Pearson's correlation coefficient. 
Table 1. Body weight, food intake, fluid intake, liver weight, and skeletal muscle weight of SAMP8 mice.

\begin{tabular}{|c|c|c|c|c|}
\hline & \multicolumn{4}{|c|}{ Ethanol } \\
\hline & $0 \%$ (Control) & $1 \%$ & $2 \%$ & $5 \%$ \\
\hline Final body wt (g) & $32.8 \pm 0.8^{1}$ & $34.7 \pm 1.9$ & $33.8 \pm 1.2$ & $32.6 \pm 0.9$ \\
\hline Gains in body wt ( $\mathrm{g}$ for $14 \mathrm{wk}$ ) & $5.1 \pm 0.3$ & $6.6 \pm 1.0$ & $6.3 \pm 0.8$ & $4.5 \pm 0.5$ \\
\hline Liver $(g)$ & $1.58 \pm 0.05$ & $1.48 \pm 0.09$ & $1.54 \pm 0.06$ & $1.51 \pm 0.05$ \\
\hline Gastrocnemius muscle (g) & $0.144 \pm 0.007$ & $0.143 \pm 0.005$ & $0.136 \pm 0.005$ & $0.148 \pm 0.006$ \\
\hline Total food intake (g for $14 \mathrm{wk}$ ) & $540^{2}$ & 497 & 530 & 475 \\
\hline Total fluid intake (g for $14 \mathrm{wk}$ ) & 757 & 663 & 817 & 637 \\
\hline Ethanol intake (g for14 wk) & 0 & 5.3 & 13.1 & 25.5 \\
\hline \multicolumn{5}{|c|}{ Ethanol intake $\left(\mathrm{g} \cdot \mathrm{kg}\right.$ body weight $\left.{ }^{-1} \cdot \mathrm{d}^{-1}\right)$} \\
\hline at $14 \mathrm{wk}$-old & 0 & 1.38 & 3.92 & 7.50 \\
\hline at $18 \mathrm{wk}$-old & 0 & 1.16 & 3.11 & 5.91 \\
\hline at 22 wk-old & 0 & 1.19 & 2.29 & 5.62 \\
\hline
\end{tabular}

${ }^{1}$ Values are mean \pm SE $(n=10)$, and unaffected by ethanol intake ( $p>0.05$, Dunnett's test).

${ }^{2}$ Because the 5 mice were kept together in a cage, the average of the 2 cages data of food intake, fluid intake and ethanol intake in each group (10 mice per group) was indicated.

\section{RESULTS}

Growth, food intake, and fluid intake

Final body weight, food intake, fluid intake, liver weight, and gastrocnemius weight were unaffected by ethanol consumption ( $p>0.05$; Table 1). Based on fluid intake and body weight data, daily ethanol ingestion for the $1 \%, 2 \%$, and $5 \%$ ethanol groups was approximately $1.2-1.4,2.3-3.9$, and $5.6-7.5 \mathrm{~g} \cdot \mathrm{kg}^{-1} \cdot \mathrm{d}^{-1}$, respectively (Table 1).

Senescence grading scores

The senescence grading scores of total, skin and hair, and spine categories at 18 and $23 \mathrm{wk}$ are shown in Fig. 1. Compared to the control group $(0 \%$ ethanol in water), the $1 \%$ ethanol group had significantly lower total (Fig. 1A), skin and hair (Fig. 1B), and spine (Fig. 1C) senescence grading scores (Dunnett's test, $p<0.05$ ) at 18 and $23 \mathrm{wk}$, whereas the $5 \%$ ethanol group had significantly lower spine senescence grading scores at these time points (Dunnett's test, $p<0.05$ ). Consumption of $2 \%$ ethanol caused no effects on these scores. The senescence grading scores of the behavior and eyes categories were unaffected by ethanol consumption $(p>$ 0.05 , data not shown). Overall, the $1 \%$ ethanol group exhibited larger reductions in senescence grading scores than the $2 \%$ and $5 \%$ ethanol groups.

Limb and forearm grip strength

Compared with the control group, only the $1 \%$ ethanol group had an $18 \%$ greater limb grip strength at 21 wk (Dunnett's test, $p<0.05$; Fig. 2A). There was no effect of ethanol consumption on the limb grip strength at $17 \mathrm{wk}$ and on the forearm grip strength at 17 and 21 wk ( $p>0.05$; Fig. 2B). The total senescence score at $23 \mathrm{wk}$ was not correlated with the limb and forearm grip strength at $21 \mathrm{wk}(r=-0.356$ and -0.451 , respectively, $p>0.05$ ).

Plasma metabolites

Peaks for 185 metabolites were detected, and concen-
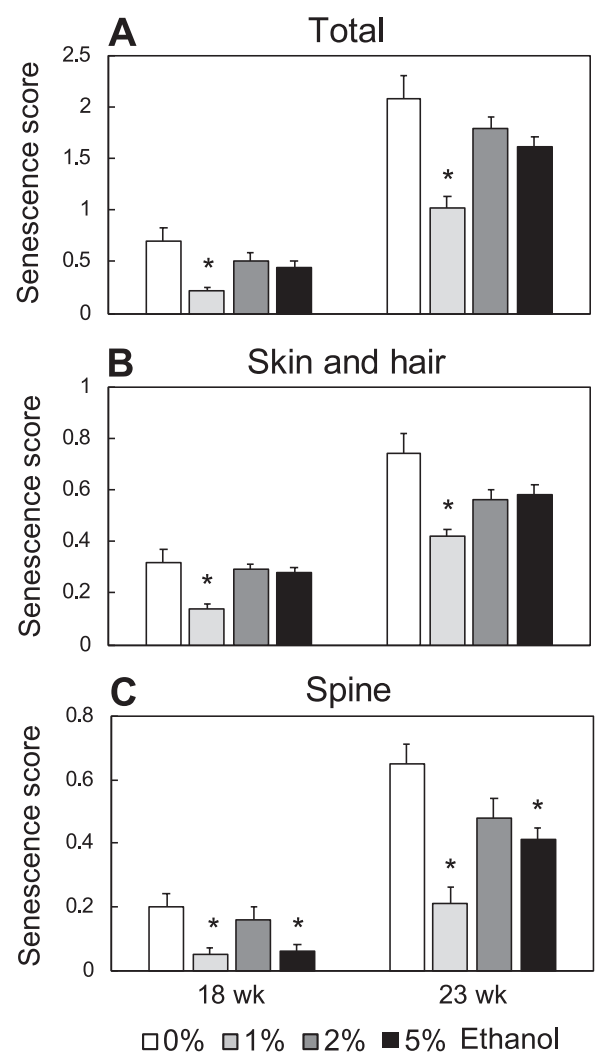

Fig. 1. Effects of ethanol intake on senescence grading scores in SAMP8 mice. A, total scores; B, scores of skin and hair; $\mathrm{C}$, scores of spine. Values are mean $\pm \mathrm{SE}$ $(n=10) .{ }^{*}$ Significantly different from the control $(0 \%$ ethanol) (Dunnett's test, $p<0.05$ ).

trations for 71 of them were quantified with or without using the standards. Levels of 7 metabolites were significantly affected by ethanol consumption (Dunnett's test, $p<0.05$; Fig. 3). Meanwhile, ethanol consumption tended to affect the levels of other 7 metabolites $(0.05<$ 
$p<0.10$; Supplemental Online Material, Table S1).

Anti-oxidative metabolites: $\alpha$-ketoglutarate, hippurate, pipecolate, and isethionate

Compared with the control group, the levels of $\alpha$ ketoglutarate were significantly (32\%) higher in the $2 \%$ ethanol group $(p<0.05$, Fig. $3 \mathrm{~A})$ but not in the $1 \%$ eth-
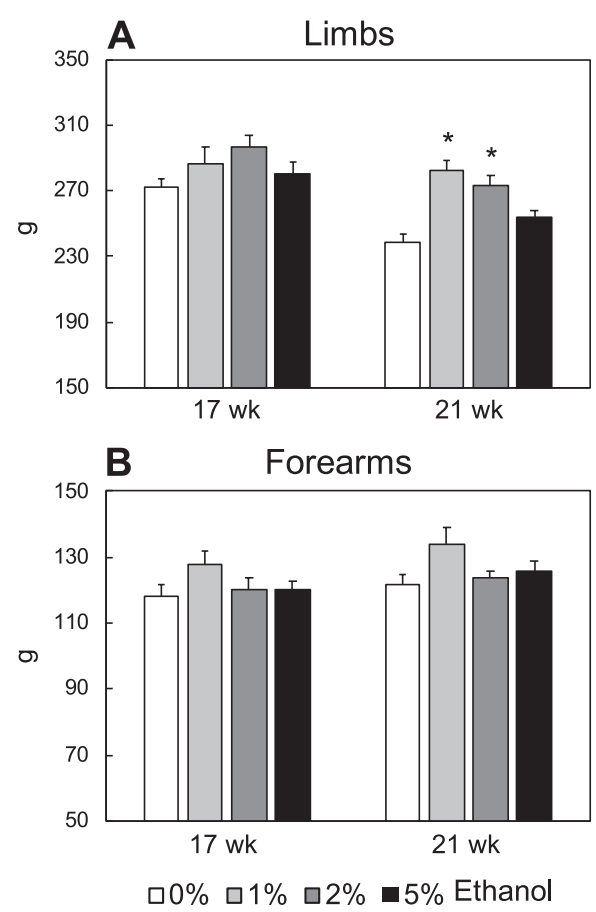

Fig. 2. Effects of ethanol intake on grip strength in SAMP8 mice. A, limbs; B, forearms. Values are mean \pm SE $(n=10)$. ${ }^{*}$ Significantly different from the control ( $0 \%$ ethanol) (Dunnett's test, $p<0.05)$. anol group $(p<0.05)$. Hippurate levels were significantly greater in the $1 \%$ and $2 \%$ ethanol groups than the control group $(37 \%$ and $69 \%$ higher, respectively, $p<0.05$; Fig. 3B). The levels of pipecolate were significantly increased by $2 \%$ ethanol $(19 \%$ higher, $p<0.05$; Fig. 3C). The levels of lysine, a precursor of pipecolate, were not affected by ethanol consumption $(p>0.10$, data not shown). Isethionate levels showed a trend of increasing with ethanol intake $(0.5<p<0.1$; Supplemental Online Material, Table S1). The levels of other antioxidant metabolites such as carnosine, homocarnosine, anserine, glutathione, and taurine were unaffected ( $p>0.10$, data not shown). In addition, the levels of $\alpha$-ketoglutarate related metabolites, such as citrate, cis-aconitate, isocitrate, succinate, and malate were also unaffected ( $p>0.10$, data not shown). The concentrations of ethanol in drinking water were significantly correlated with the levels of $\alpha$-ketoglutarate $(r=0.768)$, hippurate $(r=0.923)$, pipecolate $(r=0.664)$, and isethionate $(r=0.678)(p<0.05$ for all $)$.

Methyl donors: betaine, N,N-dimethylglycine (DMG), and methionine

The levels of betaine were significantly lower in the $1 \%$ and $2 \%$ ethanol groups than the control group (23\% and 25\% lower, respectively, $p<0.05$; Fig. 3D). Compared to the control group, DMG level was significantly lower in the $2 \%$ ethanol group (18\% lower; $p<$ 0.05 ) but not the $1 \%$ ethanol group ( $p>0.05$, Fig. 3E). The levels of ethanol in drinking water were significantly correlated with the levels of betaine $(r=-0.642)$ and DMG $(r=-0.776)(p<0.05$ for each). The level of another methyl donor, methionine, tended to be lower in the $1 \%$ and $2 \%$ ethanol groups than the control group (both $16 \%$ lower; $0.05<p<0.1$; Supplemental
A a-Ketoglutarate

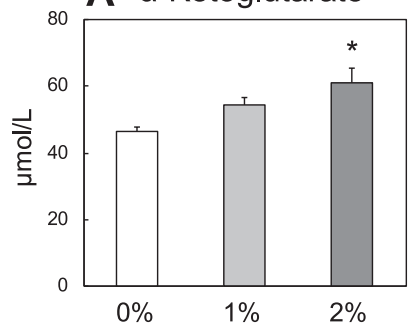

B Hippurate

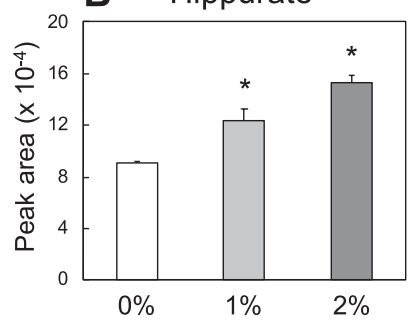

C Pipecolate

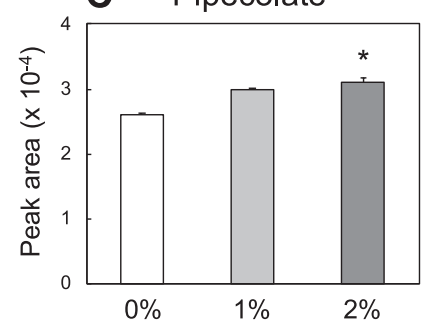

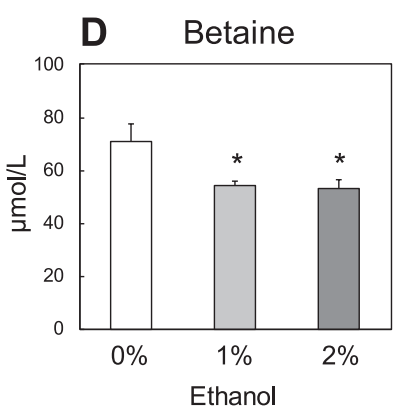
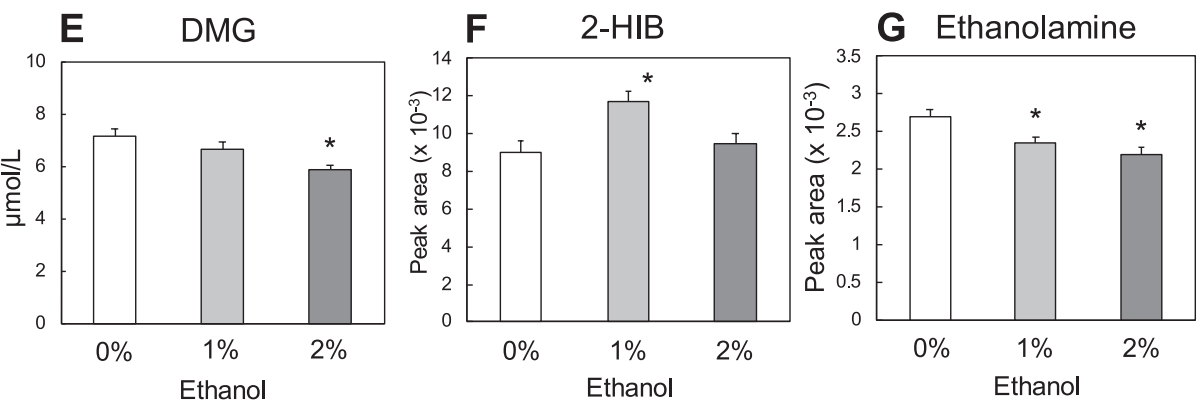

Fig. 3. Effects of ethanol intake on plasma levels of $\alpha$-ketoglutarate, hippurate, pipecolate, betaine, $N, N$-dimethylglycine (DMG), 2-hydroxyisobutyrate (2-HIB), and ethanolamine. A, $\alpha$-ketoglutarate; B, hippurate; C, pipecolate; D, betaine; E, DMG; F, 2-HIB; G, ethanolamine. Values are mean \pm SE $(n=4)$. Metabolites were quantified according to the relative peak area of each analyte to the internal standard obtained from CE-TOF/MS. * Significantly different from the control $(0 \%$ ethanol) (Dunnett's test, $p<0.05$ ). 

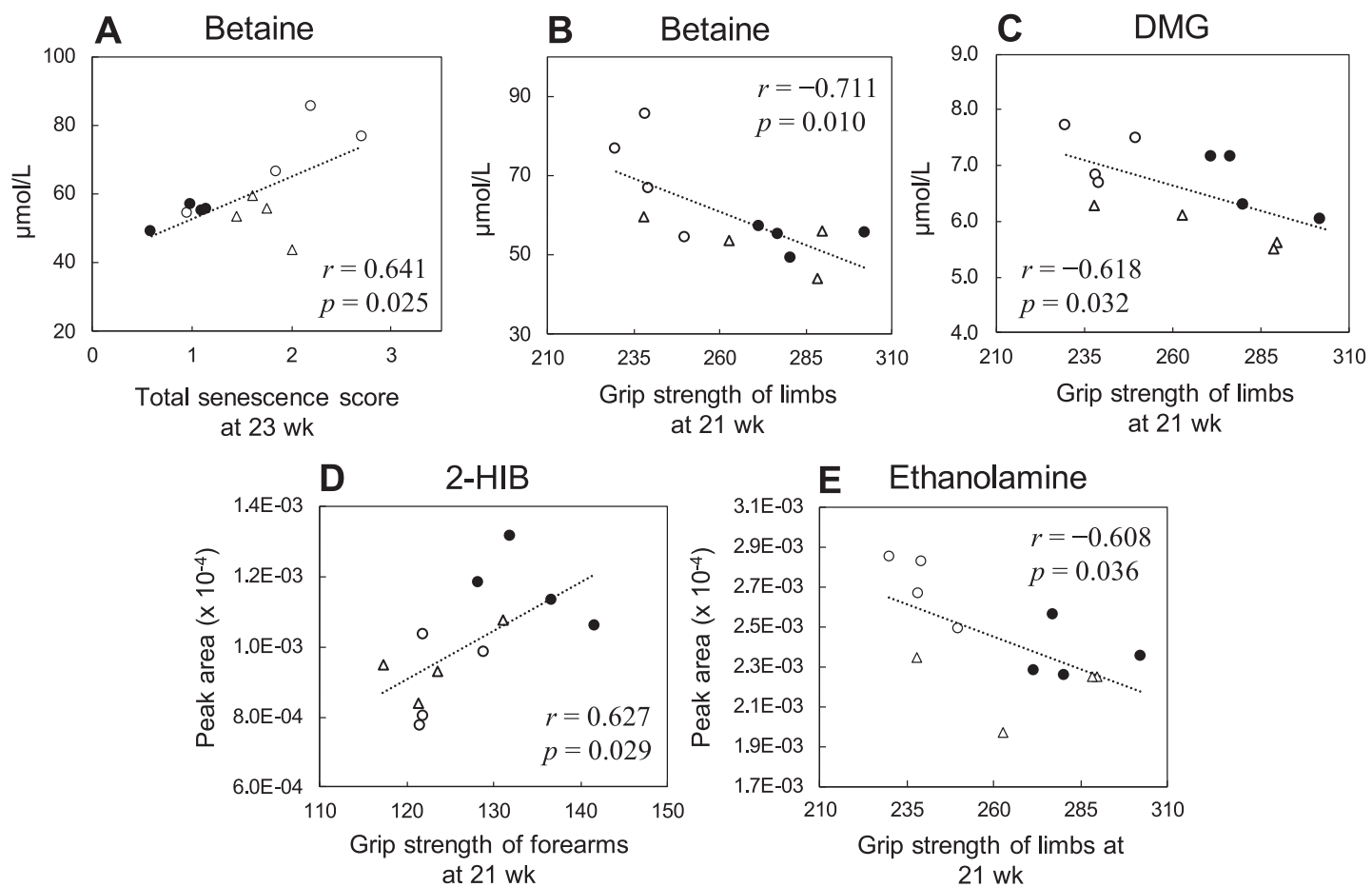

Fig. 4. Correlations of plasma metabolites with total senescence score, grip strength of limbs, and grip strength of forearms. A, betaine vs. total senescence score at $23 \mathrm{wk}$; B, betaine vs. grip strength of limbs at 21 wk; C, DMG vs. grip strength of limbs at $21 \mathrm{wk}$; D, 2-HIB vs. grip strength of forearms at $21 \mathrm{wk}$; E, ethanolamine vs. grip strength of limbs at $21 \mathrm{wk}$. Metabolites were quantified according to the relative peak area of each analyte to the internal standard obtained from CE-TOF/MS. White circle, control ( $\%$ ethanol); black circle, $1 \%$ ethanol; white triangle, $2 \%$ ethanol.

Online Material, Table S1). Other methyl donors and their related metabolites, such as sarcosine, choline, glycine, serine, glycerophosphocholine, and phosphorylcholine were not affected $(p>0.1$, data not shown).

2-Hydroxyisobutyrate (2-HIB)

The level of 2-HIB was significantly higher in the $1 \%$ ethanol group than that in the control and $2 \%$ ethanol groups $(30 \%$ and $23 \%$ higher, respectively; $p<0.05)$. The levels of ethanol in drinking water were not correlated with the level of 2-HIB $(r=0.134, p>0.05$, Fig. $3 \mathrm{~F})$.

\section{Ethanolamine}

Levels of ethanolamine in both the $1 \%$ and $2 \%$ ethanol groups were slightly lower than that in the control group (13\% and $18 \%$ lower, respectively; $p<0.05$, Fig. $3 \mathrm{G})$. The level of ethanol in drinking water was significantly correlated with the level of ethanolamine $(r=$ $-0.820, p<0.05$ ).

Other metabolites

Ethanol treatment also tended to affect the levels of other metabolites such as phenaceturate, glycerate, homovanillate, kynurenine, and $N^{6}$-methllysine $(0.05<$ $p<0.10$; Supplemental Online Material, Table S1).

Correlations of plasma metabolite levels with senescence scores and grip strength

The plasma levels of betaine were positively correlated with the total senescence grading score at $23 \mathrm{wk}$ $(r=0.641, p<0.05$; Fig. 4A). Meanwhile, the levels of betaine, DMG, and ethanolamine were inversely correlated with the limb grip strength at $21 \mathrm{wk}(r=$
$-0.711,-0.618$, and -0.608 , respectively, $p<0.05$; Fig. 4B, 4C, and 4E). The levels of 2-HIB were significantly correlated with the forearm grip strength at $21 \mathrm{wk}(r=0.627, p<0.05$; Fig. 4D), but weakly associated with limb grip strength at $21 \mathrm{wk}(r=0.513, p=$ $0.088)$ or total senescence score at $23 \mathrm{wk}(r=-0.534$, $p=0.073)$.

\section{DISCUSSION}

Consistent with findings from our previous study (8), the total senescence grading score in the $1 \%$ ethanol group was the lowest among the four treatment groups, whereas the scores of the $2 \%$ and $5 \%$ ethanol groups did not differ from those of the control. Furthermore, the present study revealed that, when compared against the control group, only mice given $1 \%$ ethanol had greater limb grip strength at $21 \mathrm{wk}$. This data suggests that $1 \%$ ethanol may be favorable for senescence and muscle strength, but not $2 \%$ and $5 \%$ ethanol, thereby supporting the J-curve hypothesis of alcohol. Of note, we previously reported that $2 \%$ ethanol stimulated spontaneous locomotor movement in SAMP8 mice, but $1 \%$ ethanol did not (8); thus, the enhanced limb grip strength observed in mice given $1 \%$ ethanol is likely to be unrelated.

The present study indicated consumption of ethanol was positively associated with the levels of antioxidants, $\alpha$-ketoglutarate and hippurate. Emerging evidence suggests that $\alpha$-ketoglutarate, an intermediary metabolite in the Krebs cycle, can be used in the therapy of several 
diseases, given its immunomodulatory, antioxidant, anti-inflammatory, and anticancer effects (16, 17). Recent studies further suggest that $\alpha$-ketoglutarate can prolong lifespan (18). $\alpha$-Ketoglutarate is involved in various fundamental processes, including collagen synthesis (19) and epigenetic regulation (20). $\alpha$-Ketoglutarate also influences several age-related processes, including stem cell proliferation (21) and osteoporosis (22). Accordingly, it is possible that low-dose ethanol might delay processes related to aging in mice, although we did not detect any significant association between $\alpha$-ketoglutarate and the total senescence score and muscle strength in our study.

Hippurate or N-benzoylglycine possesses radicalscavenging activity (23), and is a mammalian-microbial co-metabolite that originates from bacterial fermentation of dietary aromatic compounds (polyphenols, purines or aromatic amino acids) to benzoate which is further conjugated to glycine in the liver (24). Actually, the commercial pellets (MF, Oriental Yeast) used in the present study were made from corn, wheat bran, defatted soy bean, defatted rice bran, alfalfa, fish meal, defatted cow milk, soy bean oil, and beer yeast (Catalog of Oriental Yeast) and therefore contain polyphenols. There is growing evidence that hippurate is negatively associated with metabolic syndrome and Crohn's disease, and positively associated with brain health (25-29). Brial et al. found that chronic subcutaneous infusions of hippurate in mice fed a high-fat diet improved glycemic control and insulin secretion (through increased $\beta$-cell mass), as well as reduced hepatic inflammation and fibrosis (30). Collectively, the results imply that low-dose ethanol may have beneficial effect on aging mice by elevating hippurate. Recent studies suggest that low-dose ethanol inhibits oxidative stress via the upregulation of anti-oxidant systems such as cystathionine $\gamma$-lyase and alcohol dehydrogenase 1 $(8,10)$. Since low-dose ethanol elevated hippurate levels in our mice, it is presumable this would have a beneficial impact on the aforementioned conditions, warranting mechanistic studies. The levels of pipecolate was slightly, but significantly increased by $2 \%$ ethanol. Previous study suggested picolate can protect mammalian cells against oxidative stress in HEK293 cells through the mechanism involving pipecolate oxidase (31). However, other study indicated pipecolate induces oxidative stress in vitro in cerebral cortex of rats (32). Thus, at present, it is unknown if the increased pipecolate by $2 \%$ ethanol is beneficial or not.

With regards to the methyl donors, the levels of DMG decreased slightly following ethanol consumption, which corroborates with earlier, related research in humans (33). Another methyl-donor, betain levels were also lower in the ethanol groups. Of note, the levels of betaine were positively associated with senescence score at 23 wk. Additionally, the levels of betaine and DMG were inversely correlated with limb grip strength at 21 wk. Importantly, studies suggest that dietary methionine restriction extends lifespan possibly through the restriction of methyl donors (34). Therefore, intake of low-dose ethanol may be especially beneficial for aging mice, calling for further investigations. However, methyl donor deficiency is reported to have negative effects on homocysteine metabolism, intestinal differentiation and barrier function, and gene expression patterns (35). Thus, the significance of the modulation of plasma levels of methyl-donors in response to low-dose ethanol remains unclear, warranting further research.

Intriguingly, the present study showed that mice in the $1 \%$ ethanol group experienced significant increases in the levels of 2-HIB compared with those in the control and 2\% ethanol groups. Furthermore, the levels of 2-HIB were significantly associated with forearm grip strength at $21 \mathrm{wk}$, and weakly associated with total senescence score at $23 \mathrm{wk}$. This metabolite is derived from the microbial degradation of valine (36), and two studies involving healthy, young men who are moderate drinkers showed that the levels of 2-HIB in urine elevated for the first $2 \mathrm{~h}$ after acute alcohol consumption, whereas, a study of young male athletes showed that same to be elevated after an $800-\mathrm{m}$ run $(37,38)$. The levels of urinary 2-HIB in the active inflammatory bowel disease (IBD) patients were lower than those in healthy control subjects (39). 2-HIB is detected at high levels in the obese patients and undernourished mice $(40,41)$. On the other hand, a recent study suggests that histone 2-hydroxyisobutyration plays roles in glucose metabolism and aging in yeast (42). Furthermore, the extension effect of calorie restriction on yeast lifespan has been considered to be partly due to higher 2-hydroxyisobutyrylation of histone (42). Thus, emerging evidence points to 2-HIB as a biomarker of energy metabolism. As such, it is possible that consumption of $1 \%$ ethanol may be capable of modulating energy metabolism in mice. Since the response of 2-HIB to lowdose ethanol appears to be similar to the J-curve effect of alcohol, it is of interest to elucidate the implication of increased 2-HIB in the $1 \%$ ethanol group.

Mice given $1 \%$ or $2 \%$ ethanol exhibited a decrease in plasma ethanolamine levels, which correlated negatively with limb grip strength at $21 \mathrm{wk}$. Ethanolamine is known to stimulate the generation of phosphatidylethanolamines, membrane phospholipids that have a wide range of structural and functional properties, including lipid synthesis, mitochondrial biogenesis, and autophagy (43). Less is known about plasma ethanolamine, although it is suggested to be a positive marker of skeletal muscle turnover (44). Our study found no correlation between the weight of the gastrocnemius and ethanolamine level, and even a negative correlation between limb grip strength and plasma ethanolamine level, indicating more research is needed to confirm its impact, or lack thereof, on muscle tissue.

\section{CONCLUSIONS}

This study used CE-TOF/MS to show that low-dose ethanol increases plasma levels of the antioxidants $\alpha$-ketoglutarate and hippurate, the anti-inflammatory metabolite $\alpha$-ketoglutarate, and the marker of energy metabolism 2-HIB, collectively providing new insights 
for minimizing chronic disease risk. Modulation of plasma hippurate and 2-HIB, microbial co-metabolites, by low-dose ethanol is of great interest since low-dose ethanol may affect the microbial co-metabolites through mechanisms involving microbiota. Further studies are necessary to elucidate how low-dose ethanol modulates the underlying biological mechanisms in order to confirm its efficacy in health promotion.

\section{Authorship}

Research conception and design: TK, HI, and NK; experiments: CF, YY, AK, and HI; statistical analysis of the data: CF, YY, and HI; interpretation of the data: CF, YY, AK, and NK; writing of the manuscript: CF, YY, TK, and NK; CF and YY were contributed equally to this work.

\section{Disclosure of state of COI}

The authors declare that they have no conflicts of interest.

\section{Acknowledgments}

This study was financially supported in part by a Grant-in-Aid for Scientific Research from the Ministry of Education, Culture, Sports, Science and Technology of Japan, by a grant from the Brewers Association of Japan, and by the HIRAKU consortium, Hiroshima University. The authors would like to thank Enago (www. enago.jp) for English language review.

\section{Supporting information}

Supplemental online material is available on J-STAGE.

\section{REFERENCES}

1) Marmot M, Brunner E. 1991. Alcohol and cardiovascular disease: the status of the U shaped curve. BMJ 303: 565-568.

2) Xi B, Veeranki SP, Zhao M, Ma C, Yan Y, Mi J. 2017. Relationship of alcohol consumption to all-cause, cardiovascular, and cancer-related mortality in U.S. adults. J Am Coll Cardiol 70: 913-922.

3) Matsumoto A, Nagasawa Y, Yamamoto R, Shinzawa M, Hasuike Y, Kuragano T, Isaka Y, Nakanishi T, Iseki K, Yamagata K, Tsuruya K, Yoshida H, Fujimoto S, Asahi K, Moriyama T, Watanabe T. 2017. The association of alcohol and smoking with CKD in a Japanese nationwide cross-sectional survey. Hypertens Res 40: 771778.

4) Wang Y, Duan H, Yang H, Lin J. 2005. A pooled analysis of alcohol intake and colorectal cancer. Int J Clin Exp Med 8: 6878-6889.

5) Toma A, Paré G, Leong DP. 2017. Alcohol and cardiovascular disease: How much is too much? Curr Atheroscler Rep 19: 13.

6) Martin CG, Agapito VV, Obeso A, Prieto-Lloret J, Bustamante R, Castañeda J, Agapito T, Gonzalez C. 2011. Moderate ethanol ingestion, redox status, and cardiovascular system in the rat. Alcohol 45: 381-391.

7) Osaki A, Okazaki Y, Kimoto A, Izu H, Kato N. 2014. Beneficial effect of low dose of ethanol on liver function and serum urate in rats fed a high-fat diet. J Nutr Sci Vitaminol 60: 408-412.
8) Kimoto A, Izu H, Fu C, Suidasari S, Kato N. 2017. Effects of low dose of ethanol on the senescence score, brain function and gene expression in senescence-accelerated mice 8 (SAMP8). Exp Ther Med 14: 1433-1440.

9) Yang Y, Takahara K, Kumrungsee T, Kimoto A, Shimamoto F, Kato N. 2019. Consumption of low-dose of ethanol suppresses colon tumorigenesis in 1,2-dimethylhydrazine-treated rats. J Nutr Sci Vitaminol 65: 443-450.

10) McCarter KD, Li C, Li J, Xu G, Sun H. 2018. Influence of low-dose alcohol consumption on post-ischemic inflammation: Role of cystathionine $\gamma$-lyase. Alcohol 76: 81-89.

11) Melchior CL, Glasky AJ, Ritzmann RF. 1993. A low dose of ethanol impairs working memory in mice in a winshift foraging paradigm. Alcohol 10: 491-493.

12) Sakazaki F, Ogino $H$, Arakawa $T$, Okuno T, Ueno $H$. 2014. Low-dose ethanol aggravates allergic dermatitis in mice. Alcohol 48: 501-508.

13) Kimball SR, Lang CH. 2018. Mechanisms underlying muscle protein imbalance induced by alcohol. Annu Rev Nutr 38: 197-217.

14) Yoshizawa K, Okumura A, Nakashima K, Sato T, Higashi T. 2017. Role of allopregnanolone biosynthesis in acute stress-induced anxiety-like behaviors in mice. Synapse 71: e21978.

15) Soga T, Ohashi Y, Ueno Y, Naraoka H, Tomita M, Nishioka T. 2003. Quantitative metabolome analysis using capillary electrophoresis mass spectrometry. J Proteome Res 2: 488-494.

16) Liu S, He L, Yao K. 2018. The antioxidative function of alpha-ketoglutarate and its applications. Biomed Res Int 2018: 3408467.

17) He L, Li H, Huang N, Zhou X, Tian J, Li T, Wu J, Tian Y, Yin Y, Yao K. 2017. Alpha-ketoglutarate suppresses the $\mathrm{NF}-\kappa \mathrm{B}-\mathrm{mediated}$ inflammatory pathway and enhances the PXR-regulated detoxification pathway. Oncotarget $\mathbf{8}$ : 102974-102988.

18) Chin RM, Fu X, Pai MY, Vergnes L, Hwang H, Deng G, Diep S, Lomenick B, Meli VS, Monsalve GC, Hu E, Whelan SA, Wang JX, Jung G, Solis GM, Fazlollahi F, Kaweeteerawat C, Quach A, Nili M, Krall AS, Godwin HA, Chang HR, Faull KF, Guo F, Jiang M, Trauger SA, Saghatelian A, Braas D, Christofk HR, Clarke CF, Teitell MA, Petrascheck M, Reue K, Jung ME, Frand AR, Huang J. 2014. The metabolite $\alpha$-ketoglutarate extends lifespan by inhibiting ATP synthase and TOR. Nature 510: $397-$ 401.

19) Myllyharju J. 2003. Prolyl 4-hydroxylases, the key enzymes of collagen biosynthesis. Matrix Biol 22: $15-24$.

20) Ito S, D'Alessio AC, Taranova OV, Hong K, Sowers LC, Zhang Y. 2010. Role of Tet proteins in $5 \mathrm{mC}$ to $5 \mathrm{hmC}$ conversion, ES-cell self-renewal and inner cell mass specification. Nature 466: 1129-1133.

21) Carey BW, Finley LW, Cross JR, Allis CD, Thompson CB. 2015. Intracellular alpha-ketoglutarate maintains the pluripotency of embryonic stem cells. Nature 518: 413416.

22) Dobrowolski PJ, Piersiak T, Surve VV, Kruszewska D, Gawron A, Pacuska P, Håkanson R, Pierzynowski SG. 2008. Dietary alpha-ketoglutarate reduces gastrectomy-evoked loss of calvaria and trabecular bone in female rats. Scand J Gastroenterol 43: 551-558.

23) Roobinisha S, Elango S, Jothimani R, Subramanian R. 2017. Isolation of hippuric acid from buffalo urine and 
its antioxidant activity. Buffalo Bulletin 36: 531-536.

24) Williams HR, Cox IJ, Walker DG, Cobbold JF, TaylorRobinson SD, Marshall SE, Orchard TR. 2010. Differences in gut microbial metabolism are responsible for reduced hippurate synthesis in Crohn's disease. BMC Gastroenterol 10: 108. doi: 10.1186/1471-230X-10108

25) Dumas ME, Barton RH, Toye A, Cloarec O, Blancher C, Rothwell A, Fearnside J, Tatoud R, Blanc V, Lindon JC, Mitchell SC, Holmes E, McCarthy MI, Scott J, Gauguier D, Nicholson JK. 2006. Metabolic profiling reveals a contribution of gut microbiota to fatty liver phenotype in insulin-resistant mice. Proc Natl Acad Sci USA 103: 12511-12516.

26) Holmes E, Loo RL, Stamler J, Bictash M, Yap IK, Chan Q, Ebbels T, De Iorio M, Brown IJ, Veselkov KA, Daviglus ML, Kesteloot H, Ueshima H, Zhao L, Nicholson JK, Elliott P. 2008. Human metabolic phenotype diversity and its association with diet and blood pressure. Nature 453: 396-400.

27) Pallister T, Jackson MA, Martin TC, Glastonbury CA, Jennings A, Beaumont M, Mohney RP, Small KS, MacGregor A, Steves CJ, Cassidy A, Spector TD, Menni C, Valdes AM. 2017. Untangling the relationship between diet and visceral fat mass through blood metabolomics and gut microbiome profiling. Int J Obes (Lond) 41: 1106-1113.

28) Hoyles L, Fernández-Real JM, Federici M, Serino M, Abbott J, Charpentier J, Heymes C, Luque JL, Anthony E, Barton RH, Chilloux J, Myridakis A, Martinez-Gili L, Moreno-Navarrete JM, Benhamed F, Azalbert V, Blasco-Baque V, Puig J, Xifra G, Ricart W, Tomlinson C, Woodbridge M, Cardellini M, Davato F, Cardolini I, Porzio O, Gentileschi P, Lopez F, Foufelle F, Butcher SA, Holmes E, Nicholson JK, Postic C, Burcelin R, Dumas ME. 2018. Molecular phenomics and metagenomics of hepatic steatosis in non-diabetic obese women. Nat Med 24: 1070-1080.

29) Johnson KVA. 2020. Gut microbiome composition and diversity are related to human personality traits. Human Microbiome J 15: 100069.

30) Brial F, Chilloux J, Nielsen T, Vieira-Silva S, Falony G, Hoyles L, Neves AL, Rodriguez-Martinez A, Mouawad GI, Pons N, Forslund S, Le Chatelier E, Le Lay AM, Nicholson JK, Hansen T, MetaHIT Consortium, Clément K, Bork P, Dusko Ehrlich S, Raes J, Pedersen O, Gauguier D, Dumas M-E. 2019. Microbiome determinants and physiological effects of the benzoate-hippurate microbial-host co-metabolic pathway. BioRxiv doi: https://doi. org/10.1101/2019.12.15.876672.

31) Natarajan SK, Muthukrishnan E, Khalimonchuk O, Mott JL, Becker DF. 2017. Evidence for pipecolate oxidase in mediating protection against hydrogen peroxide stress. J Cell Biochem 118: 1678-1688.

32) Dalazen GR, Terra M, Jacques CED, Coelho JG, Freitas R, Mazzola PN, Dutra-Filho CS. 2014. Pipecolic acid induces oxidative stress in vitro in cerebral cortex of young rats and the protective role of lipoic acid. Metab Brain Dis 29: 175-183.

33) Harada S, Takebayashi T, Kurihara A, Akiyama M,
Suzuki A, Hatakeyama Y, Sugiyama D, Kuwabara K, Takeuchi A, Okamura T, Nishiwaki Y, Tanaka T, Hirayama A, Sugimoto M, Soga T, Tomita M. 2016. Metabolomic profiling reveals novel biomarkers of alcohol intake and alcohol-induced liver injury in communitydwelling men. Environ Health Prev Med 21: 18-26.

34) Wanders D, Ghosh S, Stone KP, Van NT, Gettys TW. 2014. Transcriptional impact of dietary methionine restriction on systemic inflammation: Relevance to biomarkers of metabolic disease during aging. Biofactors 40: 13-26.

35) Bressenot A, Pooya S, Bossenmeyer-Pourie C, Gauchotte G, Germain A, Chevaux JB, Coste F, Vignaud JM, Guéant JL, Peyrin-Biroulet L. 2013. Methyl donor deficiency affects small-intestinal differentiation and barrier function in rats. Br J Nutr 109: 667-677.

36) Li M, Wang B, Zhang M, Rantalainen M, Wang S, Zhou H, Zhang Y, Shen J, Pang X, Zhang M, Wei H, Chen Y, Lu H, Zuo J, Su M, Qiu Y, Jia W, Xiao C, Smith LM, Yang S, Holmes E, Tang H, Zhao G, Nicholson JK, Li L, Zhao L. 2008. Symbiotic gut microbes modulate human metabolic phenotypes. Proc Natl Acad Sci USA 105: $2117-$ 2122.

37) Irwin C, Mienie LJ, Wevers RA, Mason S, Westerhuis JA, van Reenen M, Reinecke CJ. 2018. GS-MS based urinary organic acid profiling reveals multiple dysregulated metabolic pathways following experimental acute alcohol consumption. Sci Rep 8: 5775. doi: 10.1038/s41598018-24128-1

38) Sun T, Wu Y, Wu X, Ma H. 2017. Metabolomic profiles investigation on athletes' urine 35 minutes after an 800-meter race. J Sports Med Phys Fitness 57: 839-849.

39) Stephens NS, Siffledeen J, Su X, Murdoch TB, Fedorak RN, Slupsky CM. 2013. Urinary NMR metabolomic profiles discriminate inflammatory bowel disease from healthy. J Crohns Colitis 7: e42-e48.

40) Calvani R, Miccheli A, Capuani G, Tomassini Miccheli A, Puccetti C, Delfini M, Iaconelli A, Nanni G, Mingrone G. 2010. Gut microbiome-derived metabolites characterize a peculiar obese urinary metabotype. Int J Obes 34: 1095-1098.

41) Preidis GA, Keaton MA, Campeau PM, Bessard BC, Conner ME, Hotez PJ. 2014. The undernourished neonatal mouse metabolome reveals evidence of liver and biliary dysfunction, inflammation, and oxidative stress. J Nutr 144: $273-281$.

42) Huang J, Luo Z, Ying W, Cao Q, Huang H, Dong J, Wu Q, Zhao Y, Qian X, Dai J. 2017. 2-Hydroxyisobutyrylation on histone H4K8 is regulated by glucose homeostasis in Saccharomyces cerevisiae. Proc Natl Acad Sci USA 114: 8782-8787.

43) Calzada E, Onguka O, Claypool SM. 2016. Phosphatidylethanolamine metabolism in health and disease. Int Rev Cell Mol Biol 321: 29-88.

44) Konz T, Santoro A, Goulet L, Bazzocchi A, Battista G, Nicoletti C, Kadi F, Ostan R, Goy M, Monnard C, Martin FP, Feige JN, Franceschi C, Rezzi S. 2019. Sex-specific associations of blood-based nutrient profiling with body composition in the elderly. Front Physiol 9: 1935. 